\title{
Retinoblastoma protein expression and its predictors in triple-negative breast cancer
}

Jaymin M. Patel $\mathbb{D}^{1,2 凶}$, Andrew Goss ${ }^{1}$, Judy E. Garber ${ }^{2,3}$, Vanda Torous ${ }^{2,4}$, Edward T. Richardson ${ }^{2,5}$, Miriam J. Haviland ${ }^{6,7}$, Michele R. Hacker 1,6,7, Gordon J. Freeman $\mathbb{i D}^{2,3}$, Tessa Nalven ${ }^{1}$, Brian Alexander ${ }^{2,8}$, Larissa Lee ${ }^{2,8}$, Laura C. Collins ${ }^{2,9}$, Stuart J. Schnitt ${ }^{2,5,10}$ and Nadine Tung ${ }^{1,2,10}$

Retinoblastoma protein $(\mathrm{Rb})$ is a product of the $R B$ tumor suppressor gene. Its expression is highly prevalent in luminal breast cancers and is critical to the success of cyclin-dependent kinase (CDK) 4/6 inhibitor therapy. Expression of Rb in triple-negative breast cancer (TNBC), tumors generally associated with basal biology, is not well known. However, heterogeneity among TNBC and presence of subtypes with luminal features are well described. The purpose of this study was to determine prevalence and predictors of Rb protein expression in BRCA1-associated and sporadic TNBCs. We studied 180 TNBC patients (70 BRCA1-associated and 110 sporadic). The clinical and pathologic features of these cases were previously assessed and reported. For this study, immunohistochemical stains for Rb were performed on tissue microarray sections. Details of treatment and outcome were abstracted from medical records. Fifty-one percent of TNBC were Rb positive ( $\geq 10 \%$ nuclei staining), and $85 \%$ of these cases had $\geq 50 \%$ nuclei staining. Rb expression was significantly associated with sporadic TNBC (71.4\% vs $49.4 \% ; p<0.001)$, androgen receptor (AR) expression ( $16.5 \%$ vs $3.4 \% ; p=0.007)$, histologic grade 1 or $2(9.9 \%$ vs $2.2 \% ; p=0.04)$, and first recurrence in bone ( $8.8 \%$ vs $1.1 \% ; p=0.03$ ). Expression of $\mathrm{p} 53$ was not associated with $\mathrm{Rb}$ expression. Expression of $\mathrm{Rb}$ in TNBC was significantly associated with sporadic TNBC, AR expression, lower histologic grade, and metastasis to bone. These observations characterize a TNBC subtype with features suggestive of luminal-like biology and the potential to benefit from CDK 4/6 inhibition.

npj Breast Cancer (2020)6:19; https://doi.org/10.1038/s41523-020-0160-4

\section{INTRODUCTION}

Triple-negative breast cancer (TNBC) is an aggressive subtype that comprises $15 \%$ of all breast cancers ${ }^{1}$. It is characterized by the absence of clinically meaningful estrogen receptor (ER), progesterone receptor, and human epidermal growth factor receptor (EGFR) 2 expression. Unfortunately, this classification fails to capture the underlying heterogeneity of TNBC observed at both clinical and molecular levels $\mathrm{s}^{2,3}$. Among the major breast cancer subtypes, TNBC has the highest rate of relapse. An improved understanding of molecular subsets is essential to refine classification, identify new therapeutic targets, inform rational combinations for clinical testing, and, in turn, individualize patient management ${ }^{4}$.

Over the past 5 years, targeted inhibition of cyclin-dependent kinase (CDK) 4/6 in combination with endocrine therapy for hormone receptor-positive metastatic breast cancer has doubled progression-free survival when compared to endocrine therapy alone $^{5-7}$. Intact retinoblastoma protein $(\mathrm{Rb})$ is considered critical for success of this combination ${ }^{8,9}$. More than $90 \%$ of hormone receptor-positive (luminal-type) breast cancers have $\mathrm{Rb}$ intact ${ }^{10}$. While there is a rationale and preclinical data to support CDK 4/6 inhibition in a subset of TNBC, it is not clear which TNBCs are most likely to respond ${ }^{11-13}$.

Among the molecular subtypes of TNBC defined by gene expression profiling, the luminal androgen receptor group (LAR) is considered to be both molecularly and clinically the most similar to hormone receptor-positive breast cancers ${ }^{14}$. The LAR subtype demonstrates high expression of the androgen receptor (AR), as well as expression of luminal cytokeratins, and comprises $10-30 \%$ of all TNBC (ref. ${ }^{3}$ ). In 2016, Tung et al. reported that certain features associated with hormone receptor-positive breast cancer, in particular lower histologic grade and older age, are predictive of AR expression in TNBC (ref. ${ }^{15}$ ). It would be of great value to identify other features common to hormone receptor-positive breast cancer in TNBC, such as Rb expression, to help identify new therapeutic targets.

Preclinical data have demonstrated palbociclib-mediated cell cycle arrest in Rb-proficient TNBC cells and attenuation of this effect by $\mathrm{Rb}$ knockdown ${ }^{13}$. There are also in vitro and in vivo data on sensitivity to CDK $4 / 6$ inhibition in LAR cells. These cells demonstrate significant differences in cell cycle dynamics, when compared to basal-like cells in single-cell analyses. Palbociclibsensitive LAR cells exit mitosis and need CDK $4 / 6$ activity to reenter the cell cycle. In contrast, palbociclib-resistant basal-like cells exit mitosis into a proliferative state independent of CDK 4/6 activity $^{11}$.

Clinical trials evaluating CDK 4/6 inhibition in TNBC are currently underway $^{16}$. Retrospective data on the frequency and predictors of $\mathrm{Rb}$ expression that may inform rational therapeutic combinations are needed. To address this, we assessed the frequency of Rb expression by immunohistochemistry $(\mathrm{IHC})$ in a previously reported cohort of primary TNBC (ref. ${ }^{15}$ ), and evaluated clinical and pathologic features predictive of $\mathrm{Rb}$ expression.

\footnotetext{
'Division of Medical Oncology, Beth Israel Deaconess Medical Center, Boston, MA, USA. ${ }^{2}$ Harvard Medical School, Boston, MA, USA. ${ }^{3}$ Division of Medical Oncology, Dana-Farber/ Brigham and Women's Cancer Center, Boston, MA, USA. ${ }^{4}$ Department of Pathology, Massachusetts General Hospital, Boston, MA, USA. ${ }^{5}$ Department of Pathology, Brigham and Women's Hospital, Boston, MA, USA. ${ }^{6}$ Department of Obstetrics \& Gynecology, Beth Israel Deaconess Medical Center, Boston, MA, USA. ${ }^{7}$ Department of Epidemiology, Harvard T. H. Chan School of Public Health, Boston, MA, USA. ${ }^{8}$ Department of Radiation Oncology, Dana-Farber/Brigham and Women's Cancer Center, Boston, MA, USA. ${ }^{9}$ Department of Pathology, Beth Israel Deaconess Medical Center, Boston, MA, USA. ${ }^{10}$ These authors contributed equally: Stuart J. Schnitt, Nadine Tung. ${ }^{\circledR}$ email: jpatel1@bidmc.harvard.edu
} 


\section{RESULTS}

$\mathrm{Rb}$ expression

Of the 180 TNBC, $51 \%$ were Rb positive ( $\geq 10 \%$ of nuclei staining for $\mathrm{Rb}$ ); of those that were $\mathrm{Rb}$ positive, $85 \%$ had $\geq 50 \%$ of the nuclei staining. Among Rb-negative TNBC, $76 \%$ had $<1 \%$ of nuclei staining.

Clinical and pathologic features

Compared with patients that were Rb-negative, patients with $\mathrm{Rb}$ positive TNBC were older (median age 48.0 vs 45.8 years), but this difference was not statistically significant $(p=0.18)$. Rb-positive TNBC were more likely to be histologic grade 1 or 2 than Rbnegative TNBC (9.9\% vs $2.2 \% ; p=0.04)$. While there was more frequent nodal involvement and higher clinical stage among patients with Rb-positive compared with Rb-negative TNBC, these differences did not reach statistical significance (see Table 1). Clinical and pathologic features stratified by $\mathrm{Rb}$ nuclear staining scores $0-3$ are shown in Supplementary Table 1.

$\mathrm{Rb}$ expression in TNBC was significantly associated with the absence of a germline $B R C A 1$ mutation and a higher likelihood of AR expression (both $p \leq 0.007)^{15}$. Of the 18 AR-positive $(A R+$ ) TNBC with available $\mathrm{Rb}$ data, $83.3 \%$ were $\mathrm{Rb}$ positive (see Table 2 ). No significant differences in luminal or basal cytokeratin staining or EGFR, programmed death-ligand 1 (PD-L1), or p53 IHC expression were noted according to $\mathrm{Rb}$ status (see Tables 2 and 3 ). Molecular features stratified by $\mathrm{Rb}$ nuclear staining scores $0-3$ are shown in Supplementary Table 2.

\section{Treatment and outcomes}

There were no significant differences in type of surgery, chemotherapy, or use of radiation between patients with and without $\mathrm{Rb}$ expression (see Table 4). Follow-up data was available for $79 \%$ of the patients; median follow-up was similar for Rbnegative (median: 12.1 years) and Rb-positive patients (median: 10.5 years; $p=0.10$; see Table 5 ). Overall number of recurrences in this cohort is small limiting interpretation. No significant differences in either the frequency of local or distant recurrences were seen between $\mathrm{Rb}$-positive and $\mathrm{Rb}$-negative patients. However, site of first distant recurrence in bone was significantly more common among patients with Rb-positive (8.8\%) than $\mathrm{Rb}$ negative $(1.1 \%)$ TNBC $(p=0.03)$. No significant difference in the incidence of brain metastasis was detected between the two groups.

\section{Co-expression of $\mathrm{Rb}$ and $\mathrm{AR}$ by $\mathrm{p} 53$ status}

As noted above, AR expression ( $\geq 10 \%$ cancer cells staining) was significantly associated with $\mathrm{Rb}$ expression ( $\geq 10 \%$ nuclear staining), whereas p53 expression (suggestive of a TP53 mutation) did not significantly correlate with either AR or Rb staining (see Table 6). Contrary to expectation, p53 staining was more common among TNBC that expressed both $A R$ and $R b$ staining than among patients that lacked both $\mathrm{AR}$ and $\mathrm{Rb}(71.4 \%$ vs $54.5 \% ; p=0.15)$; however, this difference did not reach statistical significance.

\section{DISCUSSION}

In this study of 180 TNBC, $51 \%$ showed Rb expression by IHC. Lack of a $B R C A$ mutation, lower tumor grade, and AR expression were all significantly associated with $\mathrm{Rb}$ expression. Lymph node involvement and older age were also more common among $\mathrm{Rb}$ positive than Rb-negative TNBC, although these differences did not reach statistical significance. Having a first site of distant recurrence in bone also correlated with TNBC being Rb positive.

The features that we found associated with $\mathrm{Rb}$ expression in TNBC, i.e., older age, lower histologic grade, and a propensity for bone metastases are all frequently observed in hormone receptor-
Table 1. Clinical and pathologic features at presentation by retinoblastoma protein status.

\begin{tabular}{|c|c|c|c|}
\hline & \multicolumn{2}{|c|}{ Retinoblastoma protein status } & \multirow[t]{2}{*}{$P$} \\
\hline & $\begin{array}{l}\text { Negative }(<10 \%) \\
n=89\end{array}$ & $\begin{array}{l}\text { Positive ( } \geq 10 \%) \\
n=91\end{array}$ & \\
\hline $\begin{array}{l}\text { Age at diagnosis (yrs)- } \\
\text { mean } \pm S D\end{array}$ & $45.8 \pm 9.9$ & $48.0 \pm 10.9$ & 0.18 \\
\hline BRCA status-n (\%) & & & $<0.001$ \\
\hline Carrier & $44(49.4)$ & $26(28.6)$ & \\
\hline Sporadic & $44(49.4)$ & $65(71.4)$ & \\
\hline Unknown & $1(1.1)$ & $0(0.0)$ & \\
\hline Invasive histology $-n(\%)$ & & & 0.87 \\
\hline Ductal & $81(91.0)$ & $79(86.8)$ & \\
\hline Lobular & $4(4.5)$ & $6(6.6)$ & \\
\hline Mixed & $0(0.0)$ & $0(0.0)$ & \\
\hline Metaplastic & $1(1.2)$ & $1(1.1)$ & \\
\hline Unknown & $3(3.4)$ & $5(5.5)$ & \\
\hline $\begin{array}{l}\text { Tumor size }(\mathrm{cm}) \text {-median } \\
(\text { IQR) }\end{array}$ & $1.9(1.3,2.8)$ & $2.0(1.5,3.0)$ & 0.23 \\
\hline Tumor grade-n (\%) & & & 0.04 \\
\hline 1 & $0(0.0)$ & $1(1.1)$ & \\
\hline 2 & $2(2.2)$ & $8(8.8)$ & \\
\hline 3 & $87(97.8)$ & $79(86.8)$ & \\
\hline Unknown & $0(0.0)$ & $3(3.3)$ & \\
\hline $\begin{array}{l}\text { Lymphovascular invasion } \\
-n(\%)\end{array}$ & & & 0.33 \\
\hline Present & $28(31.5)$ & $36(39.6)$ & \\
\hline Absent & $58(65.2)$ & $55(60.4)$ & \\
\hline Unknown & $3(3.4)$ & $0(0.0)$ & \\
\hline $\begin{array}{l}\text { Positive lymph nodes- } \\
n(\%)\end{array}$ & & & 0.05 \\
\hline Present & $13(14.6)$ & $30(33.0)$ & \\
\hline Absent & $27(30.3)$ & $27(29.7)$ & \\
\hline Unknown & $49(55.1)$ & $34(37.4)$ & \\
\hline T classification $-n(\%)$ & & & 0.68 \\
\hline $\mathrm{T} 1$ & $54(60.7)$ & $47(51.6)$ & \\
\hline $\mathrm{T} 2$ & $24(27.0)$ & $31(34.1)$ & \\
\hline T3 & $4(4.5)$ & $3(3.3)$ & \\
\hline $\mathrm{T} 4$ & $2(2.2)$ & $2(2.2)$ & \\
\hline Unknown & $5(5.6)$ & $8(8.8)$ & \\
\hline $\mathrm{N}$ classification $-n(\%)$ & & & 0.09 \\
\hline No & $48(53.9)$ & $40(44.0)$ & \\
\hline N1 & $25(28.1)$ & $27(29.7)$ & \\
\hline N2 & $3(3.4)$ & $12(13.2)$ & \\
\hline N3 & $3(3.4)$ & $2(2.2)$ & \\
\hline Unknown & $10(11.2)$ & $10(11.0)$ & \\
\hline AJCC stage-n (\%) & & & 0.06 \\
\hline 1 & $28(31.5)$ & $18(19.8)$ & \\
\hline 2 & $33(37.1)$ & $45(49.5)$ & \\
\hline 3 & $9(10.1)$ & $15(16.5)$ & \\
\hline 4 & $2(2.2)$ & $0(0.0)$ & \\
\hline Unknown & $17(19.1)$ & $13(14.3)$ & \\
\hline
\end{tabular}

$S D$ standard deviation, IQR interquartile range, AJCC American Joint Committee on Cancer.

aTumor size is missing for one woman in the retinoblastoma proteinnegative group. 


\begin{tabular}{|c|c|c|c|}
\hline & \multicolumn{2}{|c|}{ Retinoblastoma protein status } & \multirow[t]{2}{*}{$P$} \\
\hline & $\begin{array}{l}\text { Negative } \\
(<10 \%) n=89\end{array}$ & $\begin{array}{l}\text { Positive } \\
(\geq 10 \%) n=91\end{array}$ & \\
\hline Androgen receptor- $-n(\%)$ & & & 0.007 \\
\hline Negative/ weakly positive & $84(94.4)$ & $74(81.3)$ & \\
\hline Positive (>10\%) & $3(3.4)$ & $15(16.5)$ & \\
\hline Unknown & $2(2.2)$ & $2(2.2)$ & \\
\hline p53-n (\%) & & & 0.14 \\
\hline Negative & $36(40.4)$ & $27(29.7)$ & \\
\hline Low positive ( 1 to $<10 \%$ ) & $1(1.1)$ & $6(6.6)$ & \\
\hline Positive ( $\geq 10 \%)$ & $45(50.6)$ & $52(57.1)$ & \\
\hline Unknown & 7 (7.9) & $6(6.6)$ & \\
\hline $\begin{array}{l}\text { Epidermal growth factor } \\
\text { receptor- } n(\%)\end{array}$ & & & 0.39 \\
\hline No staining & $19(21.4)$ & $25(27.5)$ & \\
\hline$\geq 10 \%$ positive & $66(74.2)$ & $63(69.2)$ & \\
\hline Positive, unknown amount & $1(1.1)$ & $0(0.0)$ & \\
\hline Unknown & $3(3.4)$ & $3(3.3)$ & \\
\hline Cytokeratin 5/6-n (\%) & & & 0.49 \\
\hline No staining & $31(34.8)$ & $37(40.7)$ & \\
\hline$\geq 10 \%$ positive & $55(61.8)$ & $52(57.1)$ & \\
\hline Positive, unknown amount & $1(1.1)$ & $0(0.0)$ & \\
\hline Unknown & $2(2.3)$ & $2(2.2)$ & \\
\hline Cytokeratin 14-n (\%) & & & 0.82 \\
\hline No staining & $45(50.6)$ & $49(53.9)$ & \\
\hline$\geq 10 \%$ positive & $41(46.1)$ & $41(45.1)$ & \\
\hline Positive, unknown amount & $1(1.1)$ & $0(0.0)$ & \\
\hline Unknown & $2(2.3)$ & $1(1.1)$ & \\
\hline PD-L1 cancer-n (\%) & & & 0.73 \\
\hline Negative & $63(70.8)$ & $68(74.7)$ & \\
\hline Positive ( $\geq 1 \%$ ) & $22(24.7)$ & $21(23.1)$ & \\
\hline Unknown & $4(4.5)$ & $2(2.2)$ & \\
\hline
\end{tabular}

positive breast cancer, which is already known to have a relatively high propensity for $\mathrm{Rb}$ expression. Hormone receptor-positive breast cancer also has a high propensity for luminal features. The LAR subtype of TNBC, in addition to expressing luminal features, also is notable for $A R$ overexpression. Our finding that $A R$ expression in TNBC is significantly associated with $\mathrm{Rb}$ expression supports the notion of a more luminal subset of TNBC. Contrary to expectation, TNBC expressing both $\mathrm{Rb}$ and $A R$ were more often p53 positive, a feature associated with aggressive TNBC, but findings did not reach statistical significance ${ }^{17}$. These clinical and molecular findings reflect previously described heterogeneity in TNBC, and may help to define a specific subset within TNBC that is more similar to hormone-positive/luminal-type breast cancer.

Our study is one of the few to describe frequency and predictors of $\mathrm{Rb}$ expression among TNBC. In 2009, Trere et al. prospectively evaluated $\mathrm{Rb}$ expression in 518 breast cancers, of which 53 were TNBC. They found Rb expression in $62.3 \%$ of TNBC, which they stratified further by phosphorylation status ${ }^{10}$. Comparison between that study and the current study is difficult due to their use of two different antibodies and different IHC scoring methods to define Rb positivity. Our cohort was enriched for $B R C A$ mutant patients due to partial ascertainment from high-risk clinics and from an active trial program for patients with hereditary breast cancer. Given that $\mathrm{Rb}$ expression is less common among
Table 3. Molecular features at presentation by retinoblastoma protein status among a subset $(n=51)$.

\begin{tabular}{|c|c|c|c|}
\hline & \multicolumn{2}{|c|}{ Retinoblastoma protein status } & \multirow[t]{2}{*}{$P$} \\
\hline & $\begin{array}{l}\text { Negative }(<10 \%) \\
n=29\end{array}$ & $\begin{array}{l}\text { Positive ( } \geq 10 \%) \\
n=22\end{array}$ & \\
\hline Cytokeratin 7/8-n (\%) & & & 0.16 \\
\hline Negative & $1(3.5)$ & $0(0.0)$ & \\
\hline Equivocal/uninterpretable & $6(20.7)$ & $1(4.6)$ & \\
\hline Positive & $22(75.9)$ & $21(95.5)$ & \\
\hline Cytokeratin 18-n (\%) & & & 0.48 \\
\hline Negative & $1(3.5)$ & $0(0.0)$ & \\
\hline Equivocal/uninterpretable & $4(13.8)$ & $1(4.6)$ & \\
\hline Positive & $24(82.8)$ & $21(95.5)$ & \\
\hline Cytokeratin 19-n (\%) & & & 1.0 \\
\hline Negative & $1(3.5)$ & $1(4.6)$ & \\
\hline Equivocal/uninterpretable & $1(3.5)$ & $0(0.0)$ & \\
\hline Positive & 27 (93.1) & $21(95.5)$ & \\
\hline
\end{tabular}

Table 4. Treatment characteristics by retinoblastoma protein status.

\begin{tabular}{|c|c|c|c|}
\hline & \multicolumn{2}{|c|}{ Retinoblastoma protein status } & \multirow[t]{2}{*}{$P$} \\
\hline & $\begin{array}{l}\text { Negative }(<10 \%) \\
n=89\end{array}$ & $\begin{array}{l}\text { Positive ( } \geq 10 \%) \\
n=91\end{array}$ & \\
\hline Surgery-n (\%) & & & 0.56 \\
\hline Partial mastectomy & $46(51.7)$ & $44(48.4)$ & \\
\hline Mastectomy & $25(28.1)$ & $32(35.2)$ & \\
\hline Unknown & $18(20.2)$ & $15(16.5)$ & \\
\hline Chemotherapy-n (\%) & $68(76.4)$ & $74(81.3)$ & 0.40 \\
\hline Anthracycline based & $43(63.2)$ & $59(79.7)$ & \\
\hline Anthracycline + taxane & $10(14.7)$ & $6(8.1)$ & \\
\hline Taxane based & $0(0.0)$ & $1(1.4)$ & \\
\hline CMF & $11(16.2)$ & $8(10.8)$ & \\
\hline Platinum based & $1(1.5)$ & $0(0.0)$ & \\
\hline None & $3(4.4)$ & $0(0.0)$ & \\
\hline Adjuvant radiation $-n(\%)$ & & & 0.05 \\
\hline No & $2(2.2)$ & $3(3.3)$ & \\
\hline Yes & $38(42.7)$ & $54(59.3)$ & \\
\hline Unknown & $49(55.1)$ & $34(37.4)$ & \\
\hline
\end{tabular}

CMF cyclophosphamide methotrexate fluorouracil.

TNBC in BRCA mutation carriers, it is not surprising that the frequency of $\mathrm{Rb}$ expression was lower in our study (51\% vs $62 \%$, respectively).

Our findings have potentially important therapeutic implications. CDK 4/6 inhibitors, currently used only in hormone receptorpositive breast cancer, may also be beneficial for those TNBC patients that express Rb. Yamamoto et al. published in vitro and in vivo data, indicating palbociclib and the dual mammalian target of rapamycin kinase inhibitor MLN0128 showed synergistic activity in $\mathrm{Rb}+$ models of ER-negative breast cancer ${ }^{18}$. Meanwhile, Tolaney et al. are conducting a clinical trial evaluating the role of abemaciclib in patients with Rb-positive TNBC that has been actively recruiting since May 2017, and will provide additional information on the potential role of CDK $4 / 6$ inhibitors in $\mathrm{Rb}$ positive TNBC (ref. ${ }^{16}$ ). 
Table 5. Recurrence by retinoblastoma protein status.

\begin{tabular}{|c|c|c|c|}
\hline & \multicolumn{2}{|c|}{ Retinoblastoma protein status } & \multirow[t]{2}{*}{$P$} \\
\hline & $\begin{array}{l}\text { Negative } \\
(<10 \%) n=89\end{array}$ & $\begin{array}{l}\text { Positive } \\
(\geq 10 \%) n=91\end{array}$ & \\
\hline Follow-up (years)—-median (IQR) & $12.1(6.6,15.9)$ & $10.5(3.2,15.0)$ & 0.10 \\
\hline Type of surgery-n (\%) & & & 0.56 \\
\hline Breast-conserving therapy & $46(51.7)$ & $44(48.4)$ & \\
\hline Mastectomy & $25(28.1)$ & $32(35.2)$ & \\
\hline Unknown & $18(20.2)$ & $15(16.5)$ & \\
\hline Local recurrence-n (\%) & & & 0.41 \\
\hline Yes & $9(10.1)$ & $13(14.3)$ & \\
\hline No & $58(65.2)$ & $62(68.1)$ & \\
\hline Unknown & $22(24.7)$ & $16(17.6)$ & \\
\hline \multicolumn{4}{|l|}{ Site of local recurrence- $n(\%)$} \\
\hline Chest wall & $4(4.5)$ & $4(4.4)$ & 1.0 \\
\hline Scar & $0(0.0)$ & $1(1.1)$ & 1.0 \\
\hline Ipsilateral breast & $3(3.4)$ & $5(5.5)$ & 0.72 \\
\hline Lymph node & $0(0.0)$ & $0(0.0)$ & - \\
\hline Distant recurrence- $n$ (\%) & & & 0.22 \\
\hline Yes & 15 (16.9) & $24(26.4)$ & \\
\hline No & $52(58.4)$ & $51(56.0)$ & \\
\hline Unknown & $22(24.7)$ & $16(17.6)$ & \\
\hline \multicolumn{4}{|c|}{ Site of first distance recurrence- $n(\%)$} \\
\hline Bone & $1(1.1)$ & $8(8.8)$ & 0.03 \\
\hline Lung & $4(4.5)$ & $8(8.8)$ & 0.37 \\
\hline Liver & $1(1.1)$ & $5(5.5)$ & 0.21 \\
\hline Brain & $1(1.1)$ & $1(1.1)$ & 1.0 \\
\hline Nonlocal nodal group & $0(0.0)$ & $1(1.1)$ & 1.0 \\
\hline Disseminated cutaneous & $0(0.0)$ & $1(1.1)$ & 1.0 \\
\hline $\begin{array}{l}\text { Overall incidence of brain } \\
\text { metastasis }\end{array}$ & $4(4.5)$ & $5(5.5)$ & 1.0 \\
\hline
\end{tabular}

Furthermore, our results demonstrate a statistically significant relationship between $\mathrm{Rb}$ expression and AR expression in TNBC. Clinical data supporting androgen blockade as a tolerable and potentially effective treatment option in a subset of TNBC patients continues to emerge ${ }^{19,20}$. However, which patients will benefit and what drug combinations are optimal is still unclear ${ }^{21}$. As with estrogen modulation in HR-positive metastatic breast cancer, androgen blockade may benefit from the simultaneous blockade of a cooperative signaling pathway, such as CDK 4/6-Rb (ref. ${ }^{22}$ ). Liu et al. demonstrated in vitro activity of palbociclib in combination with enzalutamide in Rb-proficient and AR-positive TNBC cells ${ }^{13}$. More recently, Traina et al. reported the results from a phase II study of 118 AR-positive TNBC patients treated with enzalutamide $160 \mathrm{mg}$ daily. Of the 78 patients deemed positive for AR expression at $a \geq 10 \%$ threshold, the clinical benefit at 16 weeks was $33 \%$, median progression-free survival was 3.3 months, and median overall survival was 17.6 months $(95 \%, \mathrm{Cl}, 11.6$-not yet reached). Toxicity was minimal with fatigue as the only grade 3 symptom $^{19}$.

Overall, targeting AR in TNBC has shown promise and combining this with CDK $4 / 6$ inhibition would be a logical next step given its association with the Rb expression demonstrated in our study. Additional studies that explore the relationship of AR and $\mathrm{Rb}$ pathways in TNBC are needed. This has the potential to improve access to safe and tolerable FDA-approved oral
Table 6. Proportions of negative, low positive, and positive p53 by molecular features at presentation.

\begin{tabular}{ll} 
p53 status & $P$ \\
\hline Negative $\begin{array}{l}\text { Low } \\
\text { positive }\end{array}$ & Positive
\end{tabular}

\begin{tabular}{lrlrl}
\hline Androgen receptor- $n(\%)$ & & & 0.17 \\
$\quad$ Negative/weakly positive & $58(39.7)$ & $5(3.4)$ & $83(56.8)$ & \\
$(n=146)$ & & & & \\
$\quad$ Positive $(>10 \% ; n=17)$ & $4(23.5)$ & $1(5.9)$ & $12(70.6)$ & \\
$\quad$ Unknown $(n=4)$ & $1(25.0)$ & $1(25.0)$ & $2(50.0)$ & \\
Retinoblastoma protein- $n(\%)$ & & & & 0.07 \\
$\quad$ Negative ( $\leq 10 \% ; n=82)$ & $36(43.9)$ & $1(1.2)$ & $45(54.9)$ & \\
$\quad$ Positive $(>10 \% ; n=85)$ & $27(31.8)$ & $6(7.1)$ & $52(61.2)$ & \\
Retinoblastoma protein and & & & & 0.15 \\
androgen receptor- $n(\%)$ & & & & \\
$\quad$ Both negative $(\leq 10 \% ; n=77)$ & $34(44.2)$ & $1(1.3)$ & $42(54.5)$ & \\
$\quad$ Both positive $(>10 \% ; n=14)$ & $3(21.4)$ & $1(7.1)$ & $10(71.4)$ & \\
$\quad$ Other $(n=72)$ & $25(34.7)$ & $4(5.6)$ & $43(59.7)$ & \\
$\quad$ Unknown $(n=4)$ & $1(25.0)$ & $1(25.0)$ & $2(50.0)$ & \\
\hline
\end{tabular}

medications for a greater percentage of breast cancer patients. However, evaluating this combination in a clinical trial setting may be challenging due to limitations in eligible patients. Our study, although not designed to determine prevalence, is notable for only $8 \%$ of TNBC (14/180) that co-express both Rb and AR.

It would also be valuable to assess PI3K status among Rbpositive TNBC given data that suggest enrichment of PIK3CA mutations in AR-positive TNBC (ref. ${ }^{23}$ ). Data exist to support the combination of palbociclib with a PI3K inhibitor ${ }^{24}$. However, the clinical tolerability of this combination is unclear. In addition, expanded immune profiling that includes tumor mutation burden, albeit no correlation with PD-L1 staining in this study, would be of interest given emerging data on synergy of checkpoint inhibition with CDK $4 / 6$ inhibition $^{25}$. Lastly, whole-genome sequencing or whole-exome sequencing coupled with RNA sequencing of the 14 $\mathrm{Rb}+/ \mathrm{AR}+$ samples will be useful to better understand and characterize this subset.

The appropriate level of $\mathrm{Rb}$ nuclear staining that is clinically meaningful or predicts response to CDK $4 / 6$ inhibition remains an unanswered question. In our study, we chose to use $\geq 10 \%$ nuclear staining based on median staining intensity. However, the level of $\mathrm{Rb}$ expression by $\mathrm{IHC}$ staining that is clinically relevant will require prospective evaluation and validation. Furthermore, in the study by Traina et al., $\geq 10 \%$ AR nuclear expression by IHC had only modest positive predictive value for androgen blockade using enzalutamide. It is also unclear if AR expression using current IHC protocols is the best biomarker for predicting response to $A R$ antagonists $^{19}$.

Our study has certain limitations. In addition to its retrospective nature, selection bias for BRCA1 mutations carriers generally enriched in the high-risk clinics used for recruitment in our study limits our ability to determine the actual prevalence of $\mathrm{Rb}$ among TNBC in the general population. Furthermore, it is not clear which antibody for evaluating $\mathrm{Rb}$ expression is best. Our pathologic assessment utilized a commercially available antibody that is among a number available with no clear data to delineate, which is optimal for clinical use or which predicts response to therapeutic agents.

In conclusion, we observed Rb expression in $51 \%$ of 180 TNBC. Clinical and pathologic evaluation revealed AR expression, lower histologic grade, a lack of a germline BRCA mutation, and the 
development of bone metastases as factors associated with $\mathrm{Rb}$ expression. These findings suggest clinical-pathologic similarities between a subtype of TNBC and hormone receptor-positive breast cancer. Our findings have important implications for use of CDK 4/ 6 inhibitors alone or in combination with AR blockade in a subset of TNBC.

\section{METHODS}

Study population

The study population consists of 180 women diagnosed with stage I-III TNBC between 1989 and 2008. Eligible patients were identified in clinical databases from high-risk breast cancer clinics and an annotated Specialized Program of Research Excellence (SPORE) specimen bank at Beth Israel Deaconess Medical Center and Dana-Farber Cancer Institute.

\section{BRCA testing}

As previously reported, BRCA status was determined either by heteroduplex testing of banked research blood samples or through commercially available CLIA-approved assays ${ }^{15,26,27}$.

\section{Clinical and pathologic data}

Clinical data abstracted from medical records included age at diagnosis, clinical stage, tumor size, nodal involvement, germline BRCA status, treatment, and sites of recurrence. Pathologic features, including histologic type, Nottingham combined histologic grade, lymphovascular invasion, and lymph node involvement, were assessed on histologic sections of each tumor by the study pathologists (SJS and LCC).

\section{Immunohistochemical staining}

IHC staining and scoring of tissue microarrays (TMAs) containing three $0.6 \mathrm{~mm}$ cores from each tumor was performed as previously described ${ }^{15}$ to assess expression of cytokeratins (CK 5/6, CK 14), EGFR, AR, PD-L1, and tumor suppressor protein p53 $\left(\right.$ TP53) ${ }^{15}$. A subset consisting of the first 51 cases were also evaluated for CK7/8, CK18, and CK19.

\section{$\mathrm{Rb}$ and $\mathrm{p} 53 \mathrm{IHC}$ staining}

$\mathrm{Rb}$ staining was performed on TMA sections using a commercially available mouse monoclonal antibody to human Rb (clone G3-245, BD Biosciences, Franklin Lakes, $\mathrm{NJ}$ ) and scored as 0 ( $<1 \%$ nuclear staining), 1 ( $\geq 1 \%$ to $<10 \%$ nuclear staining), 2 ( $\geq 10 \%$ to $<50 \%$ nuclear staining), or 3 ( $\geq 50 \%$ nuclear staining). Antibody clone G3-245 was raised against a Trp-E-Rb fusion protein and recognizes an epitope spanning amino acids 332-344 (DARLFDHDKTLQ) of human Rb (pp110-114 Rb). In addition to previously reported use and validation ${ }^{28,29}$, BD Pharmingen performed western blot analysis to demonstrate binding at various states of $\mathrm{Rb}$ phosphorylation $(110-116 \mathrm{kD})^{30}$. Staining was performed in the Dana-Farber/Harvard Cancer Center Specialized Histopathology Core Laboratory, a CLIAcertified facility, using a protocol that is also used for routine standardof-care diagnostics in the clinical immunohistochemistry laboratory within the Department of Pathology at Brigham and Women's Hospital (BWH). All cases were run with a positive tumor control provided by the BWH Department of Pathology clinical laboratories, and with a tonsil control to ensure that the expected pattern of staining in normal proliferating cells (germinal center B cells and squamous epithelium) and other cell types found in reactive tonsil was observed. Although there is no consensus on what constitutes "Rb-positive" breast cancer, for our analysis, Rb-positive TNBC was defined as $\geq 10 \%$ nuclear staining. The highest staining observed among any of three TMA cores from each case determined its score. Similarly, p53 staining was performed on TMA sections using a mouse monoclonal antibody to human p53 (clone D0-1, ImmunoTech, Hostivař, Czech Republic) and scored as negative $(<1 \%$ nuclear staining), low positive ( $\geq 1 \%$ to $<10 \%$ nuclear staining), or high positive ( $\geq 10 \%$ nuclear staining).

\section{Statistical analysis}

Study data were collected and stored in REDCap. All analyses were performed with SAS 9.4 (SAS institute, Cary, NC, USA). Descriptive data are reported as mean \pm standard deviation, median (interquartile range), or proportion. Depending on distribution of data, continuous data were compared with a $t$-test or Wilcoxon rank-sum test, and categorical data were compared with $x^{2}$ or Fisher's exact tests. All tests were two-sided and a $p<0.05$ was considered statistically significant.

\section{Regulatory approval}

This study was approved by Dana-Farber/Harvard Cancer Center Institutional Review Board and Scientific Review Committee. Given minimal risk to study patients, no informed consent was required.

\section{DATA AVAILABILTY}

The data generated and analyzed during this study are described in the following data record: https://doi.org/10.6084/m9.figshare.12174099 (ref. ${ }^{31}$ ). The datasets supporting the findings of this study, are not publicly available due to restrictions in the IRB-approved protocol, and to protect research participant privacy. Data will be made available to authorized researchers who have submitted an IRB application Please contact Dr. Nadine Tung (email address: ntung@bidmc.harvard.edu), for data access requests.

Received: 19 August 2019; Accepted: 30 April 2020; Published online: 05 June 2020

\section{REFERENCES}

1. Dent, R. et al. Triple-negative breast cancer: clinical features and patterns of recurrence. Clin. Cancer Res. 13, 4429-4434 (2007).

2. Perou, C. M. et al. Molecular portraits of human breast tumours. Nature 406 747-752 (2000).

3. Lehmann, B. D. et al. Identification of human triple-negative breast cancer subtypes and preclinical models for selection of targeted therapies. J. Clin. Invest. 121, 2750-2767(2011).

4. Lehmann, B. D., Pietenpol, J. A. \& Tan, A. R. Triple-negative breast cancer: molecular subtypes and new targets for therapy. Am. Soc. Clin. Oncol. Educ. Book, 35, e31-e39 (2015).

5. Finn, R. S. et al. Palbociclib and letrozole in advanced breast cancer. N. Engl. J. Med. 375, 1925-1936, https://doi.org/10.1056/NEJMoa1607303 (2016).

6. Hortobagyi, G. N. et al. Updated results from MONALEESA-2, a phase III trial of first-line ribociclib plus letrozole versus placebo plus letrozole in hormone receptor-positive, HER2-negative advanced breast cancer. Ann. Oncol. 29, 1541-1547 (2018).

7. Johnston, S. et al. MONARCH 3 final PFS: a randomized study of abemaciclib as initial therapy for advanced breast cancer. npj Breast Cancer 5, 5 (2019).

8. Finn, R. S. et al. PD 0332991, a selective cyclin D kinase $4 / 6$ inhibitor, preferentially inhibits proliferation of luminal estrogen receptor-positive human breast cancer cell lines in vitro. Breast Cancer Res. 11, R77 (2009).

9. Flaherty, K. T. et al. Phase I, dose-escalation trial of the oral cyclin-dependent kinase 4/6 inhibitor PD 0332991, administered using a 21-day schedule in patients with advanced cancer. Clin. Cancer Res. 18, 568-576 (2012).

10. Treré, D. et al. High prevalence of retinoblastoma protein loss in triple-negative breast cancers and its association with a good prognosis in patients treated with adjuvant chemotherapy. Ann. Oncol. 20, 1818-1823 (2009).

11. Asghar, U. S. et al. Single-cell dynamics determines response to CDK4/6 inhibition in triple-negative breast cancer. Clin. Cancer Res. 23, 5561-5572 (2017).

12. $\min$, A. et al. Abstract 2318: palbociclib, a CDK4/6 inhibitor, suppresses proliferation of triple negative breast cancer. Cancer Res. 78, 2318-2318 (2018).

13. Liu, C.-Y. et al. Combination of palbociclib with enzalutamide shows in vitro activity in RB proficient and androgen receptor positive triple negative breast cancer cells. PLOS ONE 12, e0189007 (2017).

14. Santonja, A. et al. Triple negative breast cancer subtypes and pathologic complete response rate to neoadjuvant chemotherapy. Oncotarget 9, 26406-26416 (2018).

15. Tung, N. et al. Prevalence and predictors of androgen receptor and programmed death-ligand 1 in BRCA1-associated and sporadic triple-negative breast cancer. NPJ Breast Cancer 2, 16002 (2016).

16. Dana-Farber Cancer, I., Eli, L. \& Company. Abemaciclib for Patients With Retinoblastoma-Positive, Triple Negative Metastatic Breast Cancer https://ClinicalTrials. gov/show/NCT03130439 (2020).

17. Dang, D. \& Peng, Y. Roles of p53 and p16 in triple-negative breast cancer. Breast Cancer Manag. 2, 537-544 (2013).

18. Yamamoto, T., Kanaya, N., Somlo, G. \& Chen, S. Synergistic anti-cancer activity of CDK4/6 inhibitor palbociclib and dual mTOR kinase inhibitor MLN0128 in pRbexpressing ER-negative breast cancer. Breast Cancer Res. Treat. 174, 615-625 (2019). 
19. Traina, T. A. et al. Enzalutamide for the treatment of androgen receptorexpressing triple-negative breast cancer. J. Clin. Oncol. 36, 884-890 (2018).

20. Lyons, T. et al. Safety and tolerability of adjuvant enzalutamide for the treatment of early stage androgen receptor positive $(A R+)$ triple negative breast cancer. $J$. Clin. Oncol. 36, 531-531 (2018).

21. Astvatsaturyan, K., Yue, Y., Walts, A. E. \& Bose, S. Androgen receptor positive triple negative breast cancer: Clinicopathologic, prognostic, and predictive features. PLOS ONE 13, e0197827 (2018).

22. Barton, V. N., Gordon, M. A., Richer, J. K. \& Elias, A. Anti-androgen therapy in triplenegative breast cancer. Ther. Adv. Med. Oncol. 8, 305-308 (2016).

23. Guiu, S. et al. Prognostic value of androgen receptor and FOXA1 co-expression in non-metastatic triple negative breast cancer and correlation with other biomarkers. Br. J. Cancer 119, 76-79 (2018).

24. Cretella, D. et al. The anti-tumor efficacy of CDK4/6 inhibition is enhanced by the combination with $\mathrm{PI} 3 \mathrm{~K} / \mathrm{AKT} / \mathrm{mTOR}$ inhibitors through impairment of glucose metabolism in TNBC cells. J. Exp. Clin. Cancer Res. 37, 72 (2018).

25. Goel, S. et al. CDK4/6 inhibition triggers anti-tumour immunity. Nature 548 , 471-475 (2017).

26. Lee, L. J. et al. Clinical outcome of triple negative breast cancer in BRCA1 mutation carriers and noncarriers. Cancer 117, 3093-3100 (2011).

27. Tung, N. et al. Outcome of triple negative breast cancer: comparison of sporadic and BRCA1-associated cancers. Breast Cancer Res. Treat. 146, 175-182 (2014).

28. DeCaprio, J. A. et al. SV40 large tumor antigen forms a specific complex with the product of the retinoblastoma susceptibility gene. Cell 54, 275-283 (1988).

29. Cance, W. G., Brennan, M. F., Dudas, M. E., Huang, C.-M. \& Cordon-Cardo, C. Altered Expression of the Retinoblastoma Gene Product in Human Sarcomas. N. Engl. J. Med. 323, 1457-1462 (1990).

30. BD Pharmingen. Technical Data Sheet: Purified Mouse Anti-human Retinoblastoma Protein https://www.bdbiosciences.com/ds/pm/tds/554136.pdf (2011).

31. Patel, J. M. G. et al. Metadata supporting data files in the published article: retinoblastoma protein expression and its predictors in triple negative breast cancer. figshare https://doi.org/10.6084/m9.figshare.12174099 (2020).

\section{ACKNOWLEDGEMENTS}

This work was conducted with support from Harvard Catalyst | The Harvard Clinical and Translational Science Center (National Center for Advancing Translational Sciences, National Institutes of Health Award UL 1TR002541), and financial contributions from Harvard University and its affiliated academic healthcare centers. It was also supported in part by grants from the Breast Cancer research Foundation, and Dana-Farber/Harvard Cancer Center Breast SPORE (P50CA168504) and CA163125 (G.J.F.).

\section{AUTHOR CONTRIBUTIONS}

Conception and design of work (M.R.H., S.J.S., and N.T.), data acquisition (A.G., J.E.G., V.T., E.T.R., G.J.F., T.N., B.A., L.L., L.C.C., S.J.S., and N.T.), data analysis and interpretation (J.M.P., A.G., M.J.H., M.R.H., S.J.S., and N.T.), manuscript preparation (J.M.P.), and manuscript review (all authors).

\section{COMPETING INTERESTS}

L.L. has institutional funding for investigator-initiated trial and sponsored travel from AstraZeneca. G.J.F. has patents/pending royalties on the PD-1/PD-L1 pathway from Roche, Merck MSD, Bristol-Myers Squibb, Merck KGA, Boehringer Ingelheim, AstraZeneca, Dako, Leica, Mayo Clinic, and Novartis. G.J.F. has served on advisory boards for Roche, Bristol-Myers Squibb, Xios, Origimed, Triursus, iTeos, and NextPoint. J.M.P., A.G., J.E.G., V.T., E.T.R., M.J.H., M.R.H., T.N., B.A., L.C.C., S.J.S., and N.T. declare no competing interests.

\section{ADDITIONAL INFORMATION}

Supplementary information is available for this paper at https://doi.org/10.1038/ s41523-020-0160-4.

Correspondence and requests for materials should be addressed to J.M.P.

Reprints and permission information is available at http://www.nature.com/ reprints

Publisher's note Springer Nature remains neutral with regard to jurisdictional claims in published maps and institutional affiliations. Attribution 4.0 International License, which permits use, sharing, adaptation, distribution and reproduction in any medium or format, as long as you give appropriate credit to the original author(s) and the source, provide a link to the Creative Commons license, and indicate if changes were made. The images or other third party material in this article are included in the article's Creative Commons license, unless indicated otherwise in a credit line to the material. If material is not included in the article's Creative Commons license and your intended use is not permitted by statutory regulation or exceeds the permitted use, you will need to obtain permission directly from the copyright holder. To view a copy of this license, visit http://creativecommons. org/licenses/by/4.0/

(c) The Author(s) 2020 